\title{
PARASITES GOVERN THE TOPOLOGICAL PROPERTIES OF FOOD WEBS: A CONCLUSION FROM NETWORK ANALYSIS
}

\author{
CHEN, J. L. - ZHANG, W. J.* \\ School of Life Sciences, Sun Yat-sen University, Guangzhou 510275, China \\ ${ }^{*}$ Corresponding author \\ e-mail: zhwj@mail.sysu.edu.cn,wjzhang@iaees.org \\ (Received $12^{\text {th }}$ Nov 2019; accepted $23^{\text {rd }}$ Mar 2020)
}

\begin{abstract}
Parasitism has been ignored in most of the food web research. However, it has been found to play an important role in maintaining food web structure. In order to present further evidences and findings on the functionality of parasitism in food webs, this study used the methodology of network science and meta-analysis to analyze and compare topological properties of seven high-resolution marine and estuarine food webs that include free-living species only and all species with parasites respectively. The results showed that parasites significantly changed the species structure in the food webs. The number and proportion of top species declined and that of intermediate species increased once parasites were included in the food webs. The number of links and cycles, the connectance and link density of food webs significantly increased, among which most links are related to parasites and the complexity of food webs was thus enhanced. Parasitism increased food web spacing and overall clustering coefficient and food webs were made more closely connected. Parasitism was proved to have significant influence on the relative importance of species, and even to make some species crucial species in terms of food webs.
\end{abstract}

Keywords: link density, connectance, clustering coefficient, network biology, meta-analysis

\section{Introduction}

A food web is the network to describe between-species trophic relationships and energy / matter flows (Pimm et al., 1991; Kuang and Zhang, 2011; Jiang and Zhang, 2015), in which species are nodes and interacting species are connected by links. In a representative food web, plant species are at the trophic level of the first or primary producers, herbivores are at the second or primary consumer trophic level, and carnivorous animals are at the third or secondary consumer trophic level. In addition, if there are more advanced carnivores that feed on other carnivores, they will produce an even higher trophic level. Food webs are the focus of research on stability, diversity and complexity of ecosystems (Montoya and Pimm, 2006; Pascual and Dunne, 2006; Dunne et al., 2013). As self-organizing systems, they can be used to describe the nutritional relationship between species in the nature (Pimm et al., 1991; Williams and Martinez, 2000; de Araújo et al., 2017; de Araújo, 2018; Zhang, 2015, 2018). Studying food webs helps us understand the patterns of ecosystem organization and their relationship with ecological stability (Pimm, 1991; Pimm et al., 1991; Warren, 1994; McCann, 2000; Ferrarini, 2017; Wahab et al., 2017). A further objective of food web studies is to understand how they affect the functioning of ecosystems, thus further predict the environmental problems such as climate changes and species invasion (Ings et al., 2009).

The early studies of food webs began with MacArthur (1955). Major works during his period included: (1) food webs were in text and graphically expressed; (2) spatial uniformity and relationship linearity of between-species trophic relationships were assumed to analyze the stability and equilibrium of food webs. The studies during 1990s 
to 2000s have focused on general principles of link distribution. How to find general and stable patterns in food webs was one of the focuses of those studies (May, 1973; Cohen et al., 1993; Navia et al., 2010). Studies have shown that food webs, including terrestrial, freshwater and marine food webs shared some common patterns and features as (Pimm et al., 1991; Jiang and Zhang, 2015): (1) cycles were rarely found in the food web; (2) the mean of the proportion top species (T) / intermediate species (I) / basal species (B), was an invariant value (Cohen and Newman, 1985); (3) the proportion, intermediate to top species links / basal to intermediate species links / basal to top species links, was averagely an invariant; (4) link density was nearly an invariant in the food webs with fewer species, and link density would increase with the increase of species; (6) omnivores were rarely found in certain food webs; (7) compartments were rarely found; (8) for top predators, their food chain length was generally 2 or 3, other chain lengths were rarely found.

As a biological network, the food web has various topological properties, including the number of species $(S)$ and links $(L)$, connectance $\left(C ; L / S^{2}\right)$, link density $(L / S)$, cycles, chain length, etc (Kuang and Zhang, 2011; Dunne et al., 2013; Nuwagaba and Hui, 2015). Among these, the number of species and links represent species richness. Connectance and link density can be used to quantify the complexity of food webs and are thus important indicators of food web stability (Pimm and Lawton, 1980; Dunne et al., 2002; Montoya and Solé, 2003; Dunne, 2006; Montoya et al., 2006; Allesina et al., 2008; Allesina and Pascual, 2008; Zhang, 2012a,b). In addition, other topological properties such as Degree Centrality $(D C)$ and Between Centrality $(B C)$ help us measure the importance of a species in the food web (Zhang, 2018).

Unfortunately, most of the above findings have been achieved by analyzing the interactions between free-living species. Recent studies have found that parasites can profoundly affect food web properties. Parasites were found to have almost the same richness and productivity as their free-living hosts with the similar sizes and trophic levels. Studies have demonstrated that in terms of the trophic relationship, parasites had the body size ratio to their hosts that opposites the most free-living consumer-resource body size ratio, which helps them adjust the abundance of their host species. Parasites often have complex life cycles, and sometimes need to live in different host species with various body sizes. They may have the trophic specialization modes different from the free-living predators. They may be associated with their hosts in different topological positions. Their interactions with hosts can reorganize ecological communities and change the functioning of ecosystems. All these factors may lead to their difference with free-living organisms in adapting food webs and affecting food web structures. More and more evidence have been demonstrating that parasites may uniquely alter the food chain length, connectance and robustness of food webs, and thus change the topology of food webs or even the stability, interaction strength and energy flow of food webs (Anderson and May, 1978; McCallum and Dobson, 1995; Dobson et al., 2006; Wood, 2007; Allesina et al., 2008; Allesina and Pascual, 2008a,b; Kuris et al., 2008; Hechinger et al., 2011; Nedorezov, 2012; Sato et al., 2012; Dunne et al., 2013; Jiang et al., 2015; Shakil et al., 2015).

Although most of the food web studies have shown that the vulnerability at the highest trophic level is the smallest, if the parasites parasitize the species at the intermediate trophic level rather than at the lowest trophic level, those species may have the highest vulnerability to natural enemies' attack. These findings indicated that the food web without considering parasites is incomplete. Parasitic links are so important to 
ecosystem stability because they can increase the link density and nestedness (Lafferty et al., 2006a,b). Parasites are always ignored mainly due to their small sizes, and lack of cross-disciplinary collaboration, identification and quantification of parasites in food webs, and parasitology skills (Lafferty et al., 2008).

Given the importance of parasites in food webs, how to include parasites in food web studies is becoming a very important topic in recent years and findings have been achieving (Huxham et al., 1995, 1996; Marcogliese and Cone, 1997; Memmott et al, 2000; Marcogliese, 2003; Lafferty et al., 2006a,b; Hernandez and Sukhdeo, 2008; Lafferty and Kuris, 2009; Amundsen et al., 2009, 2012; Warren et al., 2010; Kuang and Zhang, 2011; Dunne et al., 2013; Jiang et al., 2015; Jiang and Zhang, 2015). However, the further confirmation of above findings and deep exploration of the impact of parasites on topological structure of different food webs is still a problem to be addressed. For example, Kuang and Zhang (2011) studied the Carpinteria Salt Marsh (CSM) food web and have reached certain conclusions, such as the changes in species structure, and the increase in the number of cycles and links, and the increase of chain length and omnivorousness if parasites have been included, etc. Nevertheless, these results have been obtained from the topological analysis on a single food web with relatively low accuracy. In addition, although ecologists acknowledge the important role that parasites play in ecological communities, but few literatures have examined whether the addition of parasites changes the crucial species in the food web. In general, the specific mechanism of influence of parasites on the topology of food webs is still unclear and needed to be further exploited.

In recent years, with the advance of food web studies, we have been obtaining more and more full and fine quantitative data (Ings et al., 2009). These data make the findings of food webs more rigorous and credible. However, many of these results appeared to be non-natural laws because the data used was incomplete and the error has thus produced (Cohen et al., 1993; Winemiller and Polis, 1996). Aiming to present further evidences on the functionality of parasitism in food webs, the present study used the methodology of network biology and meta-analysis to analyze and compare the topological properties of seven high-resolution marine and estuarine food webs that include free-living species only and all species with parasites respectively.

\section{Materials and Methods}

\section{Materials}

\section{Data sources}

The food web data used in present study were achieved from seven sources: (1) Carpinteria Salt Marsh (CSM) (Hechinger et al., 2011); (2) Estero de Punta Banda (EPB) (Hechinger et al., 2011); (3) Bahía Falsa in Bahía San Quintín (BSQ) (Hechinger et al., 2011); (4) Flensburg Fjord on the Baltic Sea (FFB) (Zander et al., 2011); (5) Otago Harbour (OHR) (Mouritsen et al., 2011); (6) Sylt tidal basin in Germany and Denmark (STB) (Thieltges et al., 2011), and (7) the Athan estuary, Aberdeenshire (YEA) (Huxham et al., 1996). For the seven high-resolution coastal estuary food webs above, two versions of each network were analyzed: one containing predators and preys only (-FREE), and the other containing all species and links including parasitoid relationships (ALL). 
These food webs are composed of basic species, free-living species and parasites. Among them, the BSQ ALL network contains 171 species, of which 52 species are parasitic. The CSM ALL network contains 165 species, of which 58 species are parasitic. There are 214 species in the EPB ALL network, of which 76 species are parasitic. FFB ALL includes 123 species, of which 46 are parasitic species. OHR ALL has 142 species, of which 19 species are parasitic. STB ALL network contains 161 species, of which 35 species are parasitic. YEA ALL contains 134 species, of which 42 species are parasitic. The raw data for each network consists of two columns, the first column is the consumer species, and the second column contains the species with the same category as the first column, but exists as predators or hosts.

\section{Data transformation}

Before making analysis, the format of the data obtained above should be transformed. First, we constructed the $n \times n$ food web matrix of $n$ species based on the predation relationship between species, in which the elements were composed of 0 or 1 . If there is the predation relationship between the two species and the column species is predator, the element is represented by 1 . Otherwise, 0 means that there is no predation between the two species. Second, after constructing the food web matrix, UCINET and Netdraw software were used to transform the ".xlsx" file in Excel to the ".net" file in Pajek. And in UCINET, the command series was used: data $\rightarrow$ data editors $\rightarrow$ matrix editor, and then ".\#\#h" format data was saved. In Netdraw, the command series was conducted: file $\rightarrow$ open $\rightarrow$ ucinet dataset $\rightarrow$ network, and the previously saved ".\#\#h" file was opened by the command series: $\rightarrow$ file $\rightarrow$ save data as $\rightarrow$ pajek $\rightarrow$ net file, and finally, we saved the data as ".net" format file for forthcoming analyses (Jiang and Zhang, 2015; Zhang, 2012a, 2018).

\section{Methods}

\section{Pajek}

Pajek is the computational platform for analyzing and visualizing complex networks that contain up to millions of nodes. A variety of methods/algorithms for network analysis are embedded in Pajek. It is mainly used to conduct global analysis on complex networks (Batagelj and Mrvar, 2004; Kuang and Zhang, 2011; Jiang and Zhang, 2015; Zhang, 2018).

\section{Network analysis}

(1) Category of species

In the network analysis, the number of species (nodes) is generally denoted by $S$. The species in the food web can be divided into top species ( $T$; i.e., the species that cannot be fed by other species), intermediate species (I; i.e., the species acting as both predators and preys), and basal species ( $B$; i.e., the species unable to feed on other species) (Pimm et al., 1991; Dunne et al., 2013).

(2) Degree analysis

The degree of a node refers to the number of nodes connected to the node (Zhang, 2012c, 2018). Degree is the most basic topological property for complex networks. In general, the greater the degree of a node is, the higher the importance of the node in the network will be. In a directed network, the degree of a node is the sum of incoming degree and outgoing degree. Food webs are directed networks. In the Pajek software, we 
can execute the command series, Net $\rightarrow$ Partions $\rightarrow$ Degree $\rightarrow$ In/Out/All under Net/Partitions/Degree menu, to achieve the incoming degree, outgoing degree and total degree.

(3) Food chain and food cycle analysis

A cycle (i.e., circuit) is a closed chain in the food web. In the food web, there are sometimes phenomena such as $A$ feeds on $B, B$ feeds on $C$, and $C$ feeds on $A$. In such a situation, a closed loop is generated among the species $A, B$ and $C$. Cannibalism is one of the food cycles. In the Pajek, the food cycles can be obtained by running the command series, Net $\rightarrow$ Count $\rightarrow$-rings $\rightarrow$ Directed $\rightarrow$ Cyclic.

(4) Link and connectance analysis

Two species (i.e., nodes) produce a link through predation relationship. The number of links in the network is represented by $L$. The link relationships are generally classified into basal species -intermediate species link $(B-I)$, intermediate species intermediate species link (I-I), basal species - top species link $(B-T)$, and intermediate species - top species link $(I-T)$.

Connectance $(C)$ refers to the percentage of links $L$ observed in the food web as a percentage of all possible connections that may be present in the web. That is, $C=L / S^{2}$ (Dunne et al., 2013). Link density refers to the ratio of the number of links in the food web to the number of total species $(L / S)$ (Zhang, 2012a,b, 2018). If link density remains the same, the more species in the food web has, the greater the connectance will be (Pimm et al., 1991).

(5) Crucial species and node centrality analysis

Every species in the food web are different in maintaining ecosystem function. It is often necessary to identify crucial species that are extremely important or have a large impact on the food web, which is also due to the consideration of species protection (Jordán, 2009). Network topology analysis plays a powerful role in quantifying the importance of species (Livi et al., 2011). Node centrality measures can be used to quantify the importance of species in food webs. In present study, degree centrality $(D C)$ and closeness centrality $(C C)$ were used (Jiang and Zhang, 2015; Zhang, 2012a, 2018) to identify crucial species. In the Pajek, degree centrality can be obtained by implementing the command series: Net $\rightarrow$ Partions $\rightarrow \mathrm{DC} \rightarrow$ All. Closeness centrality is the average shortest path between a node and other nodes (Wasserman and Faust, 1994):

$$
C C_{i}=\frac{N-1}{\sum_{j=1}^{N} d_{i j}}
$$

where $d_{i j}$ is the distance of the shortest path between node $i$ and node $j$. Generally, the larger the $C C$ value is, the smaller the average distance between the node $i$ and all other nodes will be, and the greater the importance of the node $i$ is.

(6) Triadic structure analysis

Three nodes (i.e., vertices, species) may maximally generate 16 possible structures (triadic structures) in a directed network. For example, all possible structures generated by three nodes $\mathrm{A}, \mathrm{B}$, and $\mathrm{C}$ are $003=\mathrm{A}, \mathrm{B}, \mathrm{C} ; 012=\mathrm{A} \rightarrow \mathrm{B}, \mathrm{C} ; 102=\mathrm{A} \leftrightarrow \mathrm{B}, \mathrm{C}$; $021 \mathrm{D}=\mathrm{A} \leftarrow \mathrm{B} \rightarrow \mathrm{C} ; \quad 021 \mathrm{U}=\mathrm{A} \rightarrow \mathrm{B} \leftarrow \mathrm{C} ; \quad 021 \mathrm{C}=\quad \mathrm{A} \rightarrow \mathrm{B} \rightarrow \mathrm{C} ; \quad 111 \mathrm{D}=\mathrm{A} \leftrightarrow \mathrm{B} \leftarrow \mathrm{C} ;$ $111 \mathrm{U}=\mathrm{A} \leftrightarrow \mathrm{B} \rightarrow \mathrm{C} ; 030 \mathrm{~T}=\mathrm{A} \rightarrow \mathrm{B} \leftarrow \mathrm{C}, \mathrm{A} \rightarrow \mathrm{C} ; 030 \mathrm{C}=\mathrm{A} \leftarrow \mathrm{B} \leftarrow \mathrm{C}, \mathrm{A} \rightarrow \mathrm{C} ; 201=\mathrm{A} \leftrightarrow \mathrm{B} \leftrightarrow \mathrm{C} ;$ $120 \mathrm{D}=\mathrm{A} \leftarrow \mathrm{B} \rightarrow \mathrm{C}, \quad \mathrm{A} \leftrightarrow \mathrm{C} ; \quad 120 \mathrm{U}=\mathrm{A} \rightarrow \mathrm{B} \leftarrow \mathrm{C}, \quad \mathrm{A} \leftrightarrow \mathrm{C} ; \quad 120 \mathrm{C}=\mathrm{A} \rightarrow \mathrm{B} \rightarrow \mathrm{C}, \quad \mathrm{A} \leftrightarrow \mathrm{C} ;$ $210=A \rightarrow B \leftrightarrow C, A \leftrightarrow C ; 300=A \leftrightarrow B \leftrightarrow C, A \leftrightarrow C$ (Wasserman, 1977). However, four 
possible structures exist in the undirected network, i.e., 003, 102, 201, and 300, in which the structure 300 is a complete network called triadic structure. The number of triadic structures can be used to reflect the spacing degree of a food web. The effect of parasites on the food web spacing can be understood by comparing the numbers of triadic structures that contain free-living species and all species respectively. Since the food web is a directed network, in Pajek we can analyze triadic structures by implementating the command series, Net $\rightarrow$ Transform $\rightarrow$ Arcs-Edges $\rightarrow$ All,Info $\rightarrow$ Network $\rightarrow$ Triadic Census, or Nets $\rightarrow$ Fragment (First in Second) $\rightarrow$ Find.

(7) Clustering coefficient and similarity analysis

The clustering coefficient refers to the proportion of closed dual chains occupying the network (Dunne et al., 2013), which reflects the closeness of node connections in the network. The clustering coefficient of a node can be represented by the ratio of the observed number of links existing between adjacent nodes of the node and the total number of possible existing links. The clustering coefficient of the entire network can be achieved implementing the command series in Pajek: Net $\rightarrow$ Vector $\rightarrow$ Clustering coefficients $\rightarrow \mathrm{CC} 1$. In addition, if two nodes share more adjacent nodes, the two nodes can be considered to have the higher similarity. In present study, we used the command series in Pajek to conduct hierarchical clustering analysis and, to compare the similarity between food webs with or without parasites, and to further understand whether parasites have any influence on the similarity of species in food webs: Cluster $\rightarrow$ Create Full Cluster $\rightarrow$ Operations $\rightarrow$ Dissimilarity $\rightarrow$ Network based $\rightarrow$ Dl $\rightarrow$ All .

\section{Results}

\section{Tropic structure of food webs}

According to statistic survey, the number and proportion of top, basal and intermediate species for seven food webs, including the complete food webs with parasites (BSQ-ALL, EPB-ALL, etc.), and the food webs containing free-living species only (BSQ-FREE, EPB-FREE, etc.) are shown in Table 1.

From Table 1, we can find that the inclusion of parasites in each food web does not change the number of basal species. For instance, there are always four basal species in YEA-ALL and YEA-FREE, however, the number and proportion of intermediate and top species change greatly in all food webs. In general, the inclusion of parasites has greatly reduced the proportion of top species. For example, the number of top species in YEA reduced from 31 and 13, and the proportion reduced from $33.70 \%$ and $9.70 \%$; the number of top species in STB decreased from 27 and 11, and the proportion decreased from $21.43 \%$ and $6.83 \%$. These changes may have been due to the increase in the complexity of food web after the inclusion of parasites. In this process, some of the original top species were parasitized, and their role in the food web changed from the top species to the intermediate species. At the same time, the parasites that were temporarily the top species had the possibility of being preyed by other species, and transformed to intermediate species from the top species. As a consequence, the number of intermediate species increased and the number of top species declined drastically. Nevertheless, there were some exceptions. For instance, in the CSM food web, we can find that the number of species did not change although the species composition has changed, and the proportion increased correspondingly. This result is different from the previous research. It may be due to that other species joined the food web through migration or invasion during the later period (e.g., the number of species of free-living 
species increased from 83 and 107), which changed the original trophic relationship in the food web, and further changed the proportion of top species. Taking the EPB food web as an example (Figures 1 and 2), it is obvious that the inclusion of parasites significantly led to the reduction of top species (from 9 and 3 species), which in turn changed the proportional relationship in the food web.

Table 1. Number and proportion of top, basal and intermediate species in the seven food webs ${ }^{*}$

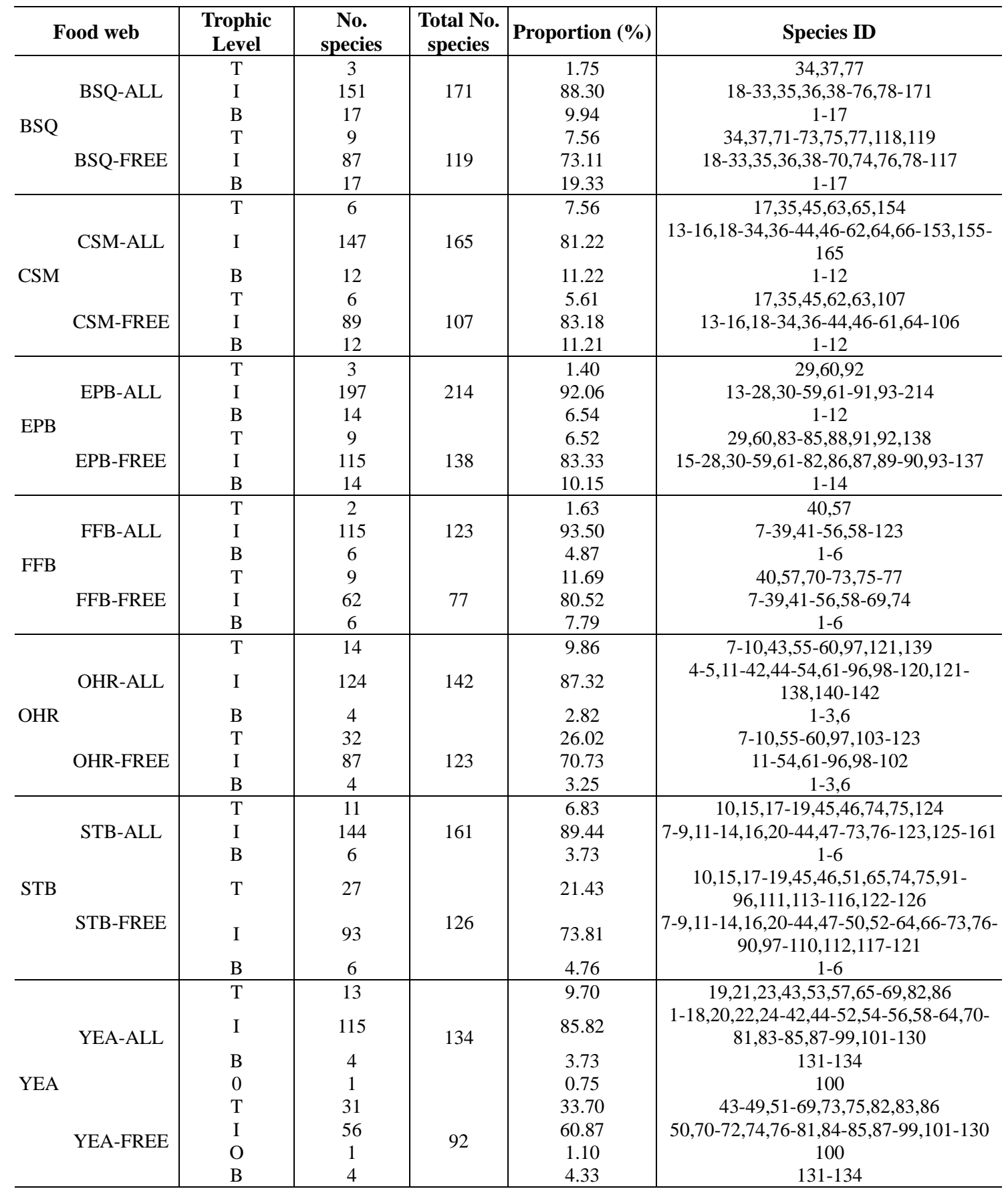

"Sources of food webs: (1) CSM (Hechinger et al., 2011); (2) EPB (Hechinger et al., 2011); (3) BSQ (Hechinger et al., 2011); (4) FFB (Zander et al., 2011); (5) OHR (Mouritsen et al., 2011); (6) STB (Thieltges et al., 2011); (7) YEA (Huxham et al., 1996) 


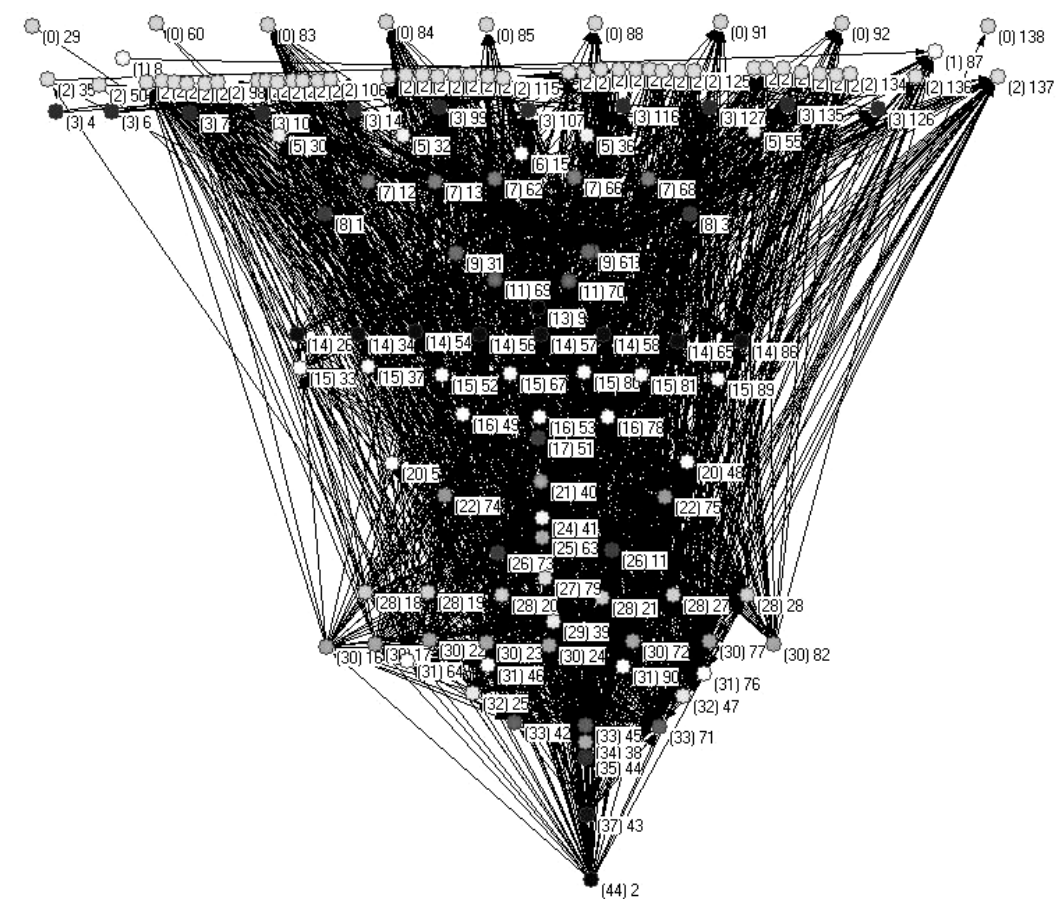

Figure 1. The EPB-ALL food web. Each node in the figure represents a different species. The nodes are arranged in the order from the smallest to the largest outgoing degree, and the outgoing degree of nodes in the same layer are the same. Top species are all at the top layer of the food web

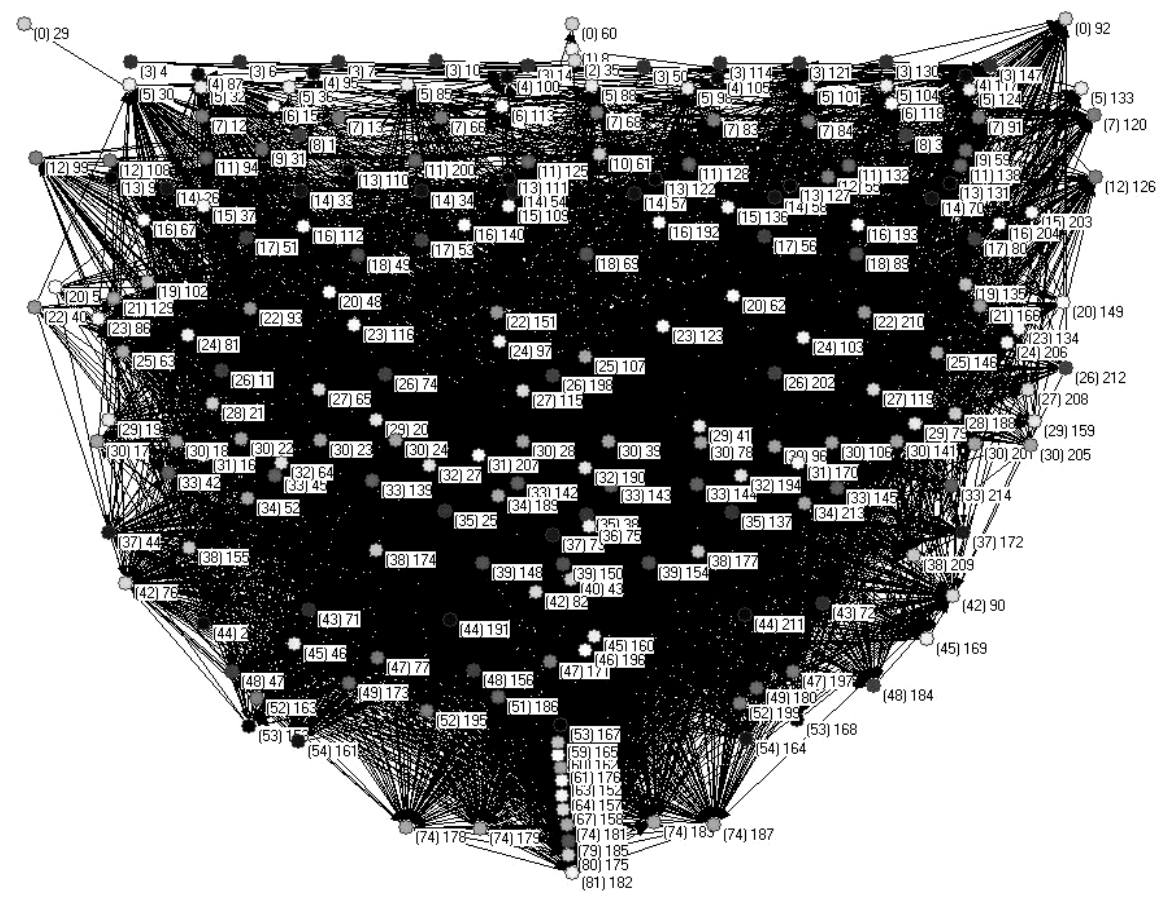

Figure 2. The EPB-FREE food web. Each node in the figure represents a different species. The nodes are arranged in the order from the smallest to the largest outgoing degree, and the outgoing degree of nodes in the same layer are the same. Top species are all at the top layer of the food web 


\section{Link analysis}

There are in total of 1192 links (i.e., connections) in the predator-prey food web of BSQ-FREE. The connectance and link density of the predator-prey food web of BSQ-FREE are 0.08 and 10.02, respectively (Figure 3). For the BSQ-ALL, the total number of links, connectance and link density are 3889, 0.13 , and 22.74 , respectively. In addition, the total number of species in BSQ-FREE accounts for 0.696 (69.6\%) of BSQ-ALL species, while the total number of links of the former is 0.307 only. Further details of link and connectance statistics are indicated in Table 2, and Figures 3 and 4.

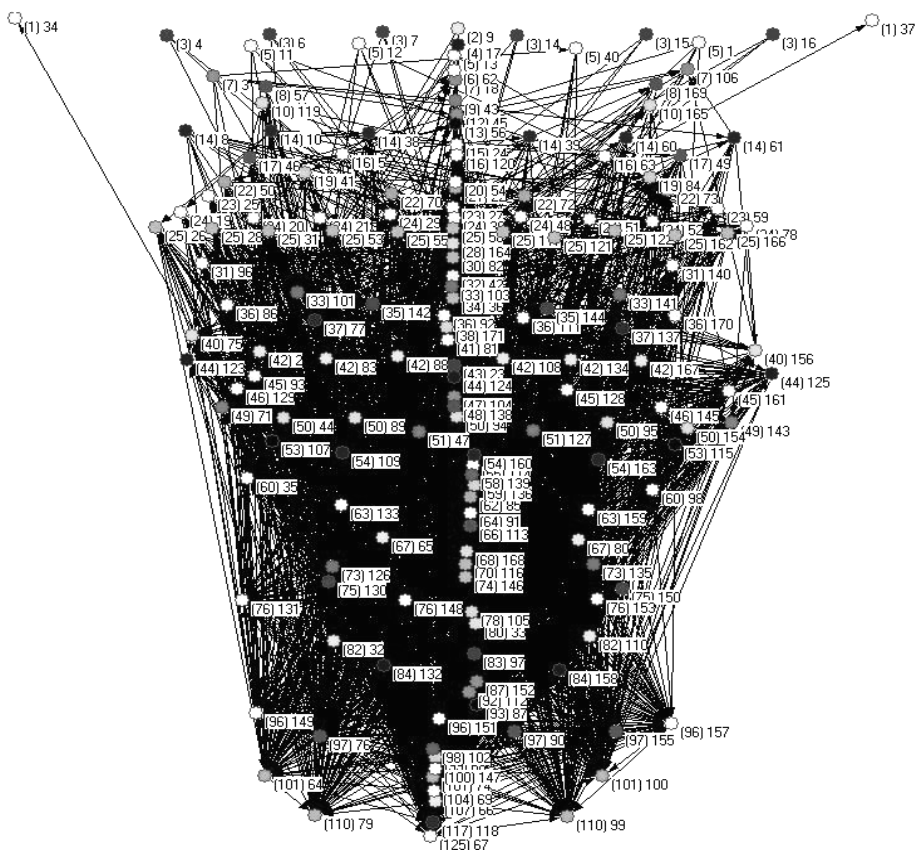

Figure 3. The BSQ-ALL food web. The number outside the brackets is species ID and the number inside the brackets is total links of the species. From top layer to bottom layer, the species are arranged in the order from the smallest to the greatest number of links. There is the same number of links for the species at the same layer

Table 2 indicates that in exception of FFB, the total number of links, connectance and link density of other free-living food webs increased significantly after parasites have been included, because the number of species in the predator-prey food webs accounts for nearly two-thirds of the complete web but the proportion of total links is rarely greater than one-third. Taking YEA as an example, the total number of YEA-FREE species accounts for $68.66 \%$ of YEA-ALL, while the proportion of total links is $33.34 \%$ only. Therefore, the inclusion of parasites has greatly enriched the trophic relationships in the food web and led the links in the food web, which increased the complexity of the food web.

\section{Food cycles}

Pimm et al. (1991) demonstrated that food cycles seldom occurred in most of the food webs. Conversely, our results indicated that food cycles frequently occurred in the food webs above, including the food webs without parasites. The inclusion of parasites may greatly increase cycles in the food web (Table 3). 
Table 2. Link and connectance statistics of the seven food webs"

\begin{tabular}{|c|c|c|c|c|c|c|c|}
\hline \multicolumn{2}{|c|}{ Food web } & No. species & $\begin{array}{l}\text { Proportion of } \\
\text { species }\end{array}$ & $\begin{array}{l}\text { Total } \\
\text { links }\end{array}$ & $\begin{array}{c}\text { Proportion of } \\
\text { links }\end{array}$ & Connectance & $\begin{array}{c}\text { Link } \\
\text { density }\end{array}$ \\
\hline \multirow{2}{*}{ BSQ } & BSQ-ALL & 171 & \multirow{2}{*}{0.696} & 3889 & \multirow{2}{*}{0.307} & 0.132998 & 22.743 \\
\hline & BSQ-FREE & 119 & & 1192 & & 0.084175 & 10.017 \\
\hline \multirow{2}{*}{ CSM } & CSM-ALL & 165 & \multirow{2}{*}{0.648} & 3850 & \multirow{2}{*}{0.278} & 0.141414 & 23.333 \\
\hline & CSM-FREE & 107 & & 1068 & & 0.093283 & 9.981 \\
\hline \multirow{2}{*}{ EPB } & EPB-ALL & 214 & \multirow{2}{*}{0.645} & 5859 & \multirow{2}{*}{0.305} & 0.127937 & 27.379 \\
\hline & EPB-FREE & 138 & & 1785 & & 0.09373 & 12.935 \\
\hline \multirow{2}{*}{ FFB } & FFB-ALL & 123 & \multirow{2}{*}{0.623} & 1526 & \multirow{2}{*}{0.429} & 0.100866 & 12.407 \\
\hline & FFB-FREE & 77 & & 655 & & 0.110474 & 8.506 \\
\hline \multirow{2}{*}{ OHR } & OHR-ALL & 142 & \multirow{2}{*}{0.867} & 1957 & \multirow{2}{*}{0.677} & 0.097054 & 13.781 \\
\hline & OHR-FREE & 123 & & 1325 & & 0.08758 & 10.772 \\
\hline \multirow{2}{*}{ STB } & STB-ALL & 161 & \multirow{2}{*}{0.783} & 3157 & \multirow{2}{*}{0.372} & 0.121793 & 19.609 \\
\hline & STB-FREE & 126 & & 1174 & & 0.073948 & 9.317 \\
\hline \multirow{2}{*}{ YEA } & YEA-ALL & 134 & \multirow{2}{*}{0.687} & 1512 & \multirow{2}{*}{0.334} & 0.084206 & 11.284 \\
\hline & YEA-FREE & 92 & & 505 & & 0.059664 & 5.489 \\
\hline
\end{tabular}

"Sources of food webs: (1) CSM (Hechinger et al., 2011); (2) EPB (Hechinger et al., 2011); (3) BSQ (Hechinger et al., 2011); (4) FFB (Zander et al., 2011); (5) OHR (Mouritsen et al., 2011); (6) STB (Thieltges et al., 2011); (7) YEA (Huxham et al., 1996)

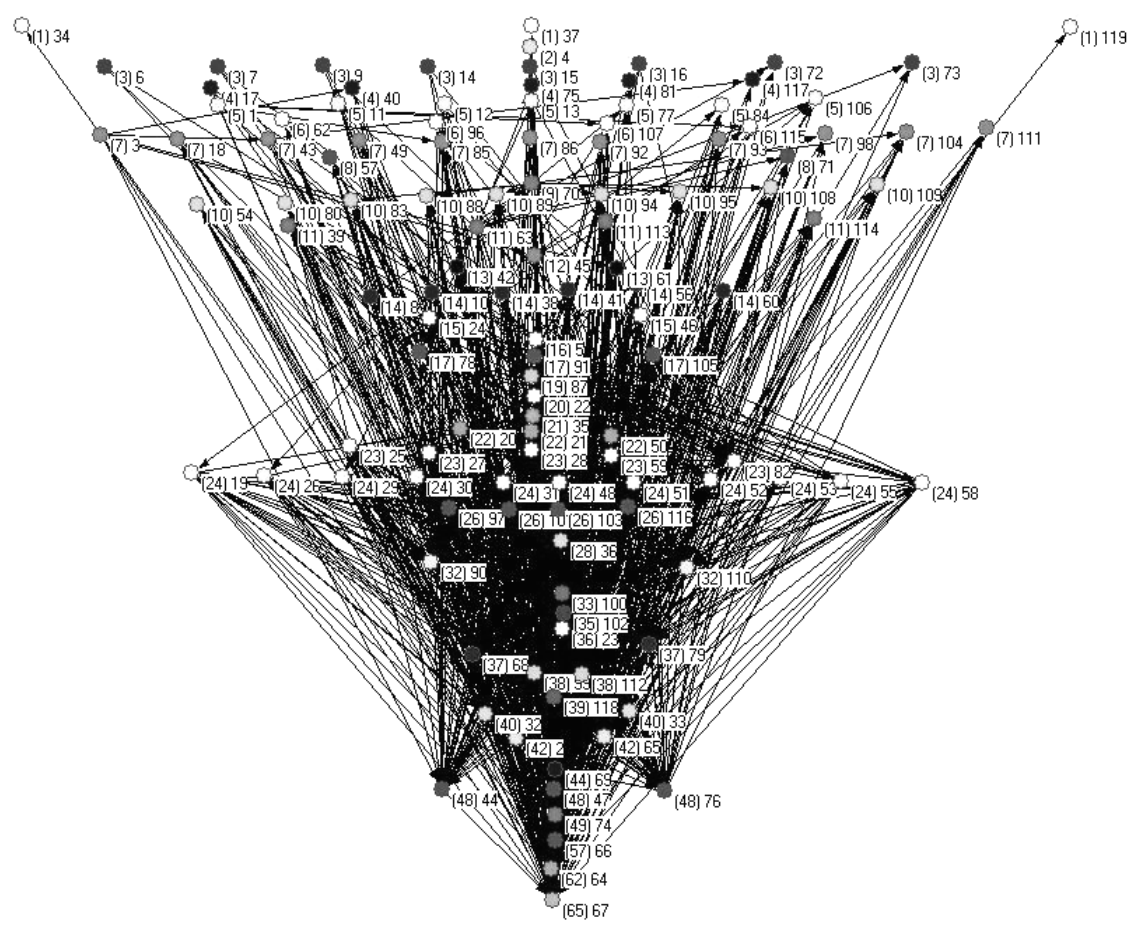

Figure 4. The BSQ-FREE food web. The number outside the brackets is species ID and the number inside the brackets is total links of the species. From top layer to bottom layer, the species are arranged in the order from the smallest to the greatest number of links. There is the same number of links for the species at the same layer 
Table 3. Number of cycles in the seven food webs ${ }^{*}$

\begin{tabular}{cc|c}
\hline & Food web & Number of cycles \\
\hline \multirow{2}{*}{ BSQ } & BSQ-ALL & 164708 \\
& BSQ-FREE & 66 \\
\hline \multirow{2}{*}{ CSM } & CSM-ALL & 166947 \\
& CSM-FREE & 176 \\
\hline \multirow{2}{*}{ EPB } & EPB-ALL & 199420 \\
& EPB-FREE & 894 \\
\hline \multirow{2}{*}{ FFB } & FFB-ALL & 8464 \\
& FFB-FREE & 0 \\
\hline \multirow{2}{*}{ OHR } & OHR-ALL & 7805 \\
& OHR-FREE & 114 \\
\hline \multirow{2}{*}{ STB } & STB-ALL & 74494 \\
& STB-FREE & 0 \\
\hline \multirow{2}{*}{ YEA } & YEA-ALL & 2844 \\
& YEA-FREE & 0 \\
\hline
\end{tabular}

"Sources of food webs: (1) CSM (Hechinger et al., 2011); (2) EPB (Hechinger et al., 2011); (3) BSQ (Hechinger et al., 2011); (4) FFB (Zander et al., 2011); (5) OHR (Mouritsen et al., 2011); (6) STB (Thieltges et al., 2011); (7) YEA (Huxham et al., 1996)

\section{Crucial species and species centrality}

From Figures 5 and 6, we can find that BSQ-ALL has more connections than BSQ-FREE, and the closeness centrality of species in BSQ slightly increases if parasites are included (BSQ-ALL). Parasitic species ID 147 shows some more importance in BSQ-ALL.

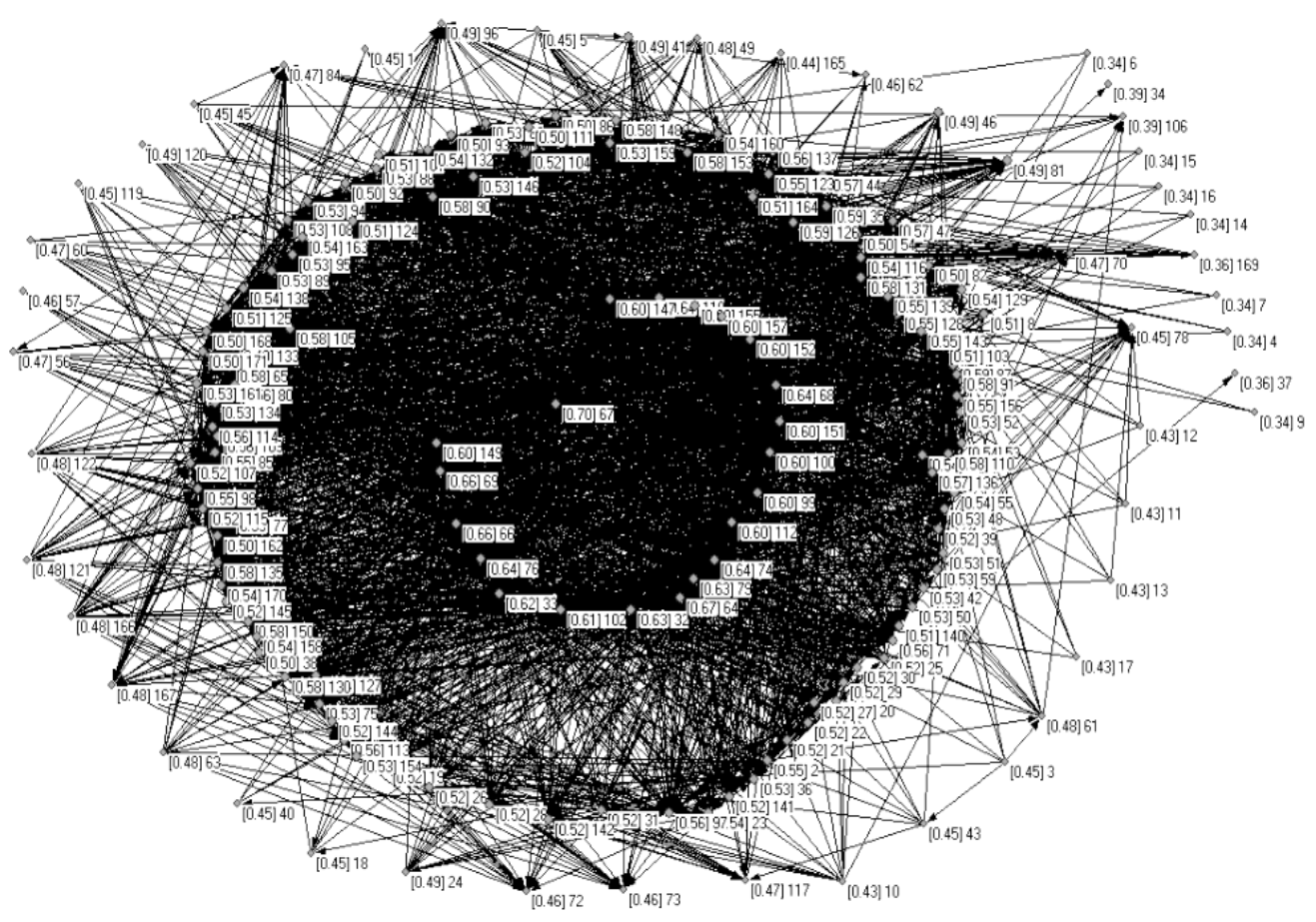

Figure 5. Closeness centrality of species in BSQ-ALL food web. The number outside the brackets is species ID and the value inside the brackets is closeness centrality of species 


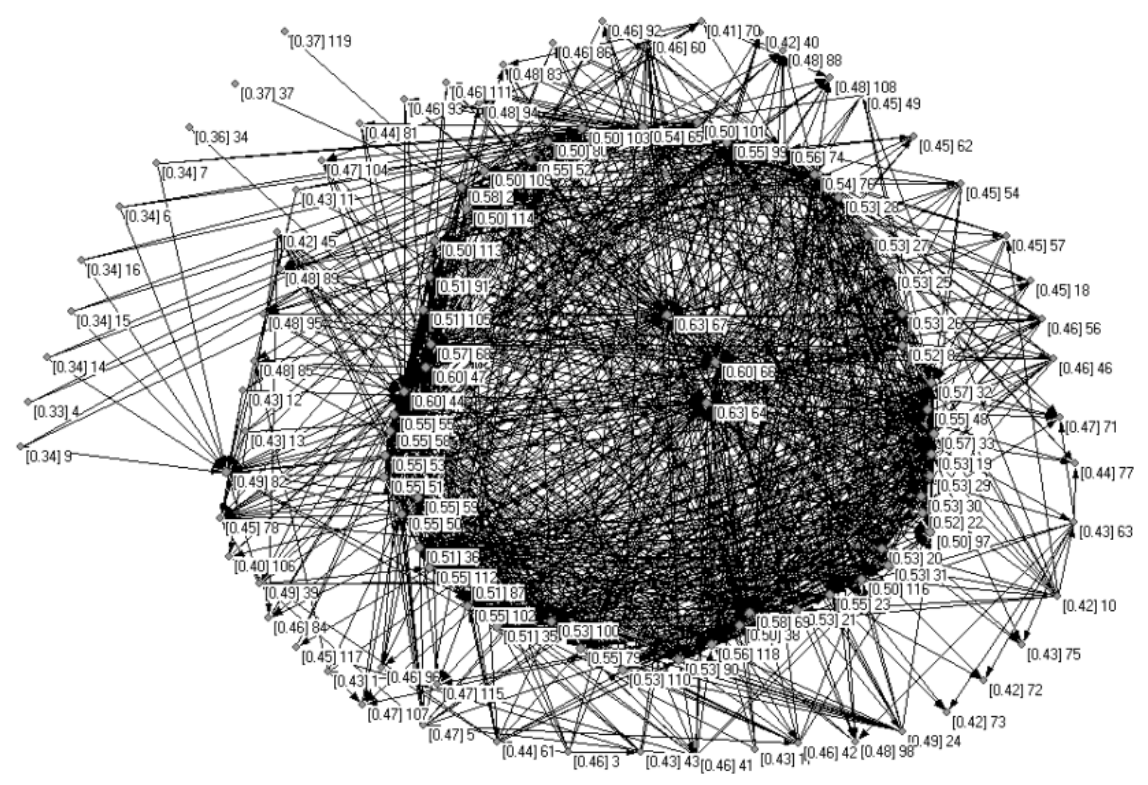

Figure 6. Closeness centrality of species in BSQ-FREE food web. The number outside the brackets is species ID and the value inside the brackets is closeness centrality of species

The species with the greater degree centrality $(D C)$ and closeness centrality $(C C)$ in the BSQ-ALL and BSQ-FREE food webs are shown in Table 4.

Table 4. The species with greater degree centrality $(D C)$ and closeness centrality $(C C$; Eq. (1)) in BSQ food web

\begin{tabular}{c|c|c|c}
\hline \multicolumn{2}{c|}{ BSQ-ALL } & \multicolumn{2}{c}{ BSQ-FREE } \\
\hline $\boldsymbol{D C}$ & $\boldsymbol{C C}$ & $\boldsymbol{D C}$ & $\boldsymbol{C C}$ \\
\hline 67 & 67 & 67 & 64 \\
118 & 64 & 64 & 67 \\
79 & 69 & 66 & 44 \\
99 & 66 & 74 & 47 \\
66 & 118 & 44 & 66 \\
69 & 76 & 47 & 69 \\
64 & 68 & 76 & 2 \\
74 & 74 & 69 & 32 \\
100 & 32 & 2 & 33 \\
147 & 79 & 65 & 68 \\
\hline
\end{tabular}

"Food web BSQ was sourced from Hechinger et al. (2011)

Since the $D C$ value of a node can generally reflect the influence of other nodes on this node, and the $C C$ value focuses on the local topology (Okamoto et al., 2008), DCs and $C C$ s in Table 4 can be combined to measure and rank nodes' importance, as indicated in Table 5. The results show that crucial species in the BSQ-ALL food web are the species IDs 67, 118, 64, 66 and 69, while the species with greater importance in the BSQ-FREE food web are the species IDs 64, 67, 44, 66 and 47. 
Table 5. Top 10 species (IDs) with greater importance in the seven food webs"

\begin{tabular}{cc|cc|cc|cc|cc|cc}
\hline \multicolumn{2}{c|}{ CSM } & \multicolumn{2}{c|}{ EPB } & \multicolumn{2}{c|}{ FFB } & \multicolumn{2}{c|}{ OHR } & \multicolumn{2}{c}{ STB } & \multicolumn{2}{c}{ YEA } \\
\hline CSM- & CSM- & EPB- & EPB- & FFB- & FFB- & OHR- & OHR- & STB- & STB- & YEA- & YEA- \\
ALL & FREE & ALL & FREE & ALL & FREE & ALL & FREE & ALL & FREE & ALL & FREE \\
\hline 51 & 34 & 72 & 72 & 75 & 75 & 5 & 2 & 60 & 60 & 85 & 43 \\
57 & 37 & 77 & 77 & 59 & 39 & 42 & 5 & 58 & 58 & 5 & 48 \\
137 & 57 & 81 & 71 & 61 & 41 & 91 & 6 & 127 & 33 & 89 & 47 \\
139 & 51 & 137 & 76 & 63 & 1 & 2 & 42 & 137 & 1 & 9 & 46 \\
143 & 50 & 76 & 59 & 39 & 4 & 125 & 22 & 133 & 4 & 77 & 59 \\
145 & 22 & 71 & 27 & 66 & 9 & 100 & 100 & 138 & 9 & 6 & 38 \\
134 & 24 & 175 & 28 & 41 & 12 & 102 & 43 & 139 & 34 & 80 & 35 \\
59 & 55 & 181 & 38 & 58 & 59 & 114 & 91 & 131 & 65 & 90 & 74 \\
55 & 21 & 185 & 42 & 71 & 15 & 6 & 75 & 131 & 33 & 32 & 92 \\
135 & 26 & 38 & 61 & 64 & 61 & 124 & 114 & 33 & 89 & 61 & 61 \\
\hline
\end{tabular}

"Sources of food webs: (1) CSM (Hechinger et al., 2011); (2) EPB (Hechinger et al., 2011); (3) BSQ (Hechinger et al., 2011); (4) FFB (Zander et al., 2011); (5) OHR (Mouritsen et al., 2011); (6) STB (Thieltges et al., 2011); (7) YEA (Huxham et al., 1996)

According to Table 5, the rank of species importance changes if the parasites are included the food web. In some food webs, parasites have the greater importance and become crucial species. For instance, there are seven parasite species in the top ten species list of STB-ALL. Parasites may have lowered the relative importance of some free-living species (e.g., the species ID 1 in FFB), or enhanced the importance of other species (e.g., the species ID 5 in YEA).

\section{Triadic structures}

Details of the triadic structures of the seven food webs are listed in Table 6.

According to Table 6, for example, FFB-ALL food web contains 3888 triadic structures while FFB-FREE food web contains 673 triadic structures only. It is obvious that the inclusion of parasites greatly increased the number of triadic structures in food webs.

Table 6. Details of triadic structures of the seven food webs*

\begin{tabular}{|c|c|c|c|}
\hline \multicolumn{2}{|c|}{ Food web } & \multirow{2}{*}{$\begin{array}{c}\text { No. triadic structures } \\
14093 \\
2081\end{array}$} & \multirow{2}{*}{\begin{tabular}{|c}
$\begin{array}{c}\text { Increase of proportion of triadic structures if } \\
\text { parasites are included }(\boldsymbol{\%})\end{array}$ \\
577.22
\end{tabular}} \\
\hline BSQ & $\begin{array}{l}\text { BSQ-ALL } \\
\text { BSQ-FREE }\end{array}$ & & \\
\hline CSM & $\begin{array}{l}\text { CSM-ALL } \\
\text { CSM-FREE }\end{array}$ & $\begin{array}{c}18423 \\
2056 \\
\end{array}$ & 796.06 \\
\hline EPB & $\begin{array}{l}\text { EPB-ALL } \\
\text { EPB-FREE }\end{array}$ & $\begin{array}{c}32916 \\
5425 \\
\end{array}$ & 506.75 \\
\hline FFB & $\begin{array}{l}\text { FFB-ALL } \\
\text { FFB-FREE }\end{array}$ & $\begin{array}{c}3888 \\
673 \\
\end{array}$ & 477.11 \\
\hline OHR & $\begin{array}{l}\text { OHR-ALL } \\
\text { OHR-FREE }\end{array}$ & $\begin{array}{l}4717 \\
2381 \\
\end{array}$ & 98.11 \\
\hline STB & $\begin{array}{l}\text { STB-ALL } \\
\text { STB-FREE }\end{array}$ & $\begin{array}{c}11379 \\
1969 \\
\end{array}$ & 477.91 \\
\hline YEA & $\begin{array}{l}\text { YEA-ALL } \\
\text { YEA-FREE }\end{array}$ & $\begin{array}{c}2816 \\
361\end{array}$ & 680.06 \\
\hline
\end{tabular}

"Sources of food webs: (1) CSM (Hechinger et al., 2011); (2) EPB (Hechinger et al., 2011); (3) BSQ (Hechinger et al., 2011); (4) FFB (Zander et al., 2011); (5) OHR (Mouritsen et al., 2011); (6) STB (Thieltges et al., 2011); (7) YEA (Huxham et al., 1996) 


\section{Clustering coefficients}

A greater clustering coefficient means the greater closeness of connections and species are thus more likely parasitized. As shown in Table 7, the clustering coefficients of each food web increase if the parasites are included.

Table 7. Clustering coefficients of the seven food webs ${ }^{*}$

\begin{tabular}{cc|c}
\hline \multicolumn{2}{|c|}{ Food web } & Clustering coefficient \\
\hline \multirow{2}{*}{ BSQ } & BSQ-ALL & 0.176856 \\
& BSQ-FREE & 0.106715 \\
\hline \multirow{2}{*}{ CSM } & CSM-ALL & 0.225561 \\
& CSM-FREE & 0.142477 \\
\hline \multirow{2}{*}{ EPB } & EPB-ALL & 0.187083 \\
& EPB-FREE & 0.169259 \\
\hline \multirow{2}{*}{ FFB } & FFB-ALL & 0.165141 \\
& FFB-FREE & 0.088927 \\
\hline \multirow{2}{*}{ OHR } & OHR-ALL & 0.140108 \\
& OHR-FREE & 0.112305 \\
\hline \multirow{2}{*}{ STB } & STB-ALL & 0.170479 \\
& STB-FREE & 0.109187 \\
\hline \multirow{2}{*}{ YEA } & YEA-ALL & 0.131878 \\
& YEA-FREE & 0.082545 \\
\hline
\end{tabular}

"Sources of food webs: (1) CSM (Hechinger et al., 2011); (2) EPB (Hechinger et al., 2011); (3) BSQ (Hechinger et al., 2011); (4) FFB (Zander et al., 2011); (5) OHR (Mouritsen et al., 2011); (6) STB (Thieltges et al., 2011); (7) YEA (Huxham et al., 1996)

\section{Conclusions}

In present study, seven high-resolution riparian food webs were used to analyze topological properties of the complete food webs (-ALL) and the food webs containing only free-living species (-FREE). The conclusions on the importance of parasites were drawn or further confirmed in the following aspects:

(1) Parasitism changes trophic structure of species in the food web, i.e., the number and proportion of top species decline and the number and proportion of intermediate species increase. Although the number of top species in the CSM-ALL and CSM-FREE food webs was the same (Kuang and Zhang, 2011), the number and proportion of intermediate species increased significantly after parasites were included, which was basically consistent with the present conclusion.

(2) The presence of parasites increases the complexity of the food web. The number of links in food webs was proved to increase significantly as the inclusion of parasites, and most of the links were related to parasites, which means that parasites play an important role in the food web and the complexity of the food web is enhanced.

(3) Parasites significantly increase the number of cycles in the food web. Pimm (1991) found that there were few cycles in the predator-predator food webs. In present study, the results of FFB, STB, and YEA food webs coincided with his conclusion. In addition, the number of cycles in the food webs increased sharply after including parasites. On the one hand, the original connection relationship became more complex and thus the possibility of cycle occurrence increased due to the existence of parasites. On the other hand, considering the complicated life cycles of parasites, a series of cycles may also be produced among parasites. 
(4) The presence of parasites alters the importance of species in the original food web. Network analysis demonstrated that parasites have produced a great impact on the relative importance of species in the food web, even as a crucial species in the complete food webs due to their great importance.

(5) Parasites increase the food web spacing. The number of triadic structures increased as the inclusion of parasites in the food web, thus making food web spacing significantly increased.

(6) Parasites increase the overall clustering coefficient of the food web, making the food web more closely connected. Parasitism is further enhanced with positive feedback mechanism as the increase of the clustering coefficient of the food web. The interaction between parasitism and the clustering coefficient is mutually reinforced.

\section{Discussion}

The food web is a major theme of fundamental ecology, which concerns with the stability, diversity and complexity of ecosystems (Pascual and Dunne, 2006). Traditional food web research mostly focuses on free-living species, while parasitism that are widespread in nature and are often difficult to detect has been ignoring (Price, 1980). As researchers become more aware of the importance of parasites and their possible effects on the structure, dynamics, and function of food webs, many have begun to focus on this potential area and a series of research have been conducting (Pascual and Dunne, 2006; Dunne et al., 2013; Michalska-Smith et al., 2018). More and more complete food webs containing parasites have been built and some research results were obtained. Studies have confirmed that parasites may change the structure and function of food webs (Sato et al., 2012). Nevertheless, the impact of parasites on the topological properties of the food web needs to be further exploited. The number of links, connectance and link density in the above food webs can reflect the potentiality of energy circulation paths, and thus may indicate the complexity of the food webs. MacArthur (1955) argued that the complexity of food webs can be considered as a key indicator of web stability. The research on the topological properties of networks also helps us measure the stability of food webs. In addition, studies have shown that parasites as pathogens of infectious diseases or as crucial species play an important role in maintaining the stability of ecological communities and ecosystems (McCallum and Dobson, 1995). Our conclusions further proved that parasites change the importance of the original species and may even become crucial species. They will increase the clustering coefficient of the food web and expand the epidemics of infectious diseases. It may be beneficial to the construction of relevant models in epidemiological studies (Shams and Khansari, 2019).

Some aspects should be further strengthened in the future studies: (1) network robustness of the food webs with parasites (Zhang, 2016), which is different from stability; (2) food web models specific to the food webs with parasites (Cohen and Newman, 1985; Cohen et al., 1990; Williams and Martinez, 2000), and (3) mechanisms of parasitism in maintaining food web structure.

Acknowledgments. This study was supported by The National Key Research and Development Program of China (2017YFD0201204) and Guangzhou Science and Technology Project (No. 201707020003). 


\section{REFERENCES}

[1] Allesina, S., Pascual, M. (2008): Network structure, predator-prey modules and stability in large food webs. - Theoretical Ecology 1: 55-64.

[2] Allesina, S., Alonso, D., Pascual, M. (2008): A general model for food web structure. Science 320(5876): 658-661.

[3] Amundsen, P. A., Lafferty, K. D., Knudsen, R., Primicerio, R., Klemetsen, A., Kuris, A. M. (2009): Food web topology and parasites in the pelagic zone of a subarctic lake. Journal of Animal Ecology 78: 563-572.

[4] Amundsen, P. A., Lafferty, K. D., Knudsen, R., Primicerio, R., Kristoffersen, R., Klemetsen, A., Kuris, A. M. (2012): New parasites and predators follow the introduction of two fish species to a subarctic lake: implications for food-web structure and functioning. - Oecologia 171: 993-1002.

[5] Anderson, R. M., May, R. M. (1978): Regulation and stability of host-parasite population interactions: I. Regulatory processes. - Journal of Animal Ecology 47: 219-247.

[6] Batagelj, V., Mrvar, A. (2004): Pajek - analysis and visualization of large networks. - In: Mutzel, P., Jünger, M., Leipert, S. (eds.) Graph Drawing GD 2001. Lecture Notes in Computer Science 2265. Springer, Berlin, Heidelberg.

[7] Cohen, J. E., Newman, C. M. (1985): A stochastic theory of community food webs: I. Models and aggregated data. - Proceedings of the Royal Society of London B Biology 224(1237): 421-448.

[8] Cohen, J. E., Luczak, T., Newman, C. M., Zhou, Z. M. (1990): Stochastic structure and nonlinear dynamics of food webs - qualitative stability in a Lotka Volterra cascade model. - Proceedings of the Royal Society of London B Biology 240: 607-627.

[9] Cohen, J. E., Pimm, S. L., Yodzis, P., Saldana, J. (1993): Body sizes of animal predators and animal prey in food webs. - Journal of Animal Ecology 62: 67-78.

[10] de Araújo, W. S., Grandez-Rios, J. M., Bergamini, L. L., Kollár, J. (2017): Exotic species and the structure of a plant-galling network. - Network Biology 7(2): 21-32.

[11] de Araújo, W. S. (2018): Robustness of plant-plant networks with different levels of habitat modification and interaction intimacy. - Network Biology 8(2): 55-64.

[12] Dobson, A. P., Lafferty, K. D., Kuris, A. M. (2006): Parasites and food webs. - Oxford University Press, Oxford: 119-135.

[13] Dunne, J. A., Williams, R. J., Martinez, N. D. (2002): Food-web structure and network theory: The role of connectance and size. - Proceedings of the National Academy of Sciences of USA 99: 12917-12922.

[14] Dunne, J. A. (2006): The network structure of food webs. - Oxford University Press, Oxford: 27-86.

[15] Dunne, J. A., Lafferty, K. D., Dobson, A. P., Hechinger, R. F., Kuris, A. M., Martinez, N. D., McLaughlin, J. P., Mouritsen, K. N., Poulin, R., Reise, K., Stouffer, D. B., Thieltges, D. W., Williams, R. J., Zander, C. D. (2013): Parasites affect food web structure primarily through increased diversity and complexity. - Plos Biology 11(6): e1001579.

[16] Ferrarini, A. (2017): A deeper insight into the equilibrium of biological and ecological networks. - Network Biology 7(4): 98-104.

[17] Hechinger, R. F., Lafferty, K. D., McLaughlin, J. P., Fredensborg, B. L., Huspeni, T. C., Lorda, J., Sandhu, P. K., Shaw, J. C., Torchin, M. E., Whitney, K. L., Kuris, A. M. (2011): Food webs including parasites, biomass, body sizes, and life stages for three California/Baja California estuaries. - Ecology 92: 791.

[18] Hernandez, A. D., Sukhdeo, M. V. K. (2008): Parasites alter the topology of a stream food web across seasons. - Oecologia 156: 613-624.

[19] Huxham, M., Raffaelli, D., Pike, A. (1995): Parasites and food web patterns. - Journal of Animal Ecology 64(2): 168-176.

[20] Huxham, M., Beaney, S., Ra Aelli, D. (1996): Do parasites reduce the chances of triangulation in a real food web? - Oikos 76(2): 284-300. 
[21] Ings, T. C., Montoya, J. M., Bascompte, J., Blüthgen, N., Brown, L., Dormann, C. F., Edwards, F., Figueroa, D., Jacob, U., Jones, J. I., Lauridsen, R. B., Ledger, M. E., Lewis, H. M., Olesen, J. M., van Veen, F. J. F., Warren, P. H., Woodward, G. (2009): Ecological networks - beyond food webs. - Journal of Animal Ecology 78(1): 253-269.

[22] Jiang, L., Zhang, W. J. (2015): Determination of keystone species in CSM food web: A topological analysis of network structure. - Network Biology 5(1): 13-33.

[23] Jiang, L., Zhang, W., Liu, G. (2015): Effects of parasitism on robustness of food webs. Selforganizology 2(2): 21-34.

[24] Jordán, F. (2009): Keystone species and food webs. - Philosophical Transactions of the Royal Society B: Biological Sciences 364(1524): 1733-1741.

[25] Kuang, W. P., Zhang, W. J. (2011): Some effects of parasitism on food web structure: a topological analysis. - Network Biology 1(3-4): 171-185.

[26] Kuris, A. M., Hechinger, R. F., Shaw, J. C., Whitney, K. L., Aguirre-Macedo, L., Boch, C. A., Dobson, A. P., Dunham, E. J., Fredensborg, B. L., Huspeni, T. C., Lorda, J., Mababa, L., Mancini, F. T., Mora, A. B., Pickering, M., Talhouk, N. L., Torchin, M. E., Lafferty, K. D. (2008): Ecosystem energetic implications of parasite and free-living biomass in three estuaries. - Nature 454: 515-518.

[27] Lafferty, K. D., Dobson, A. P., Kuris, A. M. (2006a): Parasites dominate food web links. - Proceedingsof the National Academy of Sciences of the United States of America 103(30): 11211-11216.

[28] Lafferty, K. D., Hechinger, R. F., Shaw, J. C., Whitney, K., Kuris, A. M. (2006b): Food webs and parasites in a salt marsh ecosystem. - Oxford University Press, Oxford: 119134.

[29] Lafferty, K. D., Allesina, S., Arim, M., Briggs, C. J., De Leo, G., Dobson, A. P., Dunne, J. A., Johnson, P. T. J., Kuris, A. M., Marcogliese, D. J., Martinez, N. D., Memmott, J., Marquet, P. A., McLaughlin, J. P., Mordecai, E. A., Pascual, M., Poulin, R., Thieltges, D. W. (2008): Parasites in food webs: the ultimate missing links. - Ecology Letters 11(6): 533-546.

[30] Lafferty, K. D., Kuris, A. M. (2009): Parasites reduce food web robustness because they are sensitive to secondary extinction as illustrated by an invasive estuarine snail. Philosophical Transactions of the Royal Society of London B: Biological Science 364: 1659-1663.

[31] Livi, C. M., Jordán, F., Lecca, P., Okey, T. A. (2011): Identifying key species in ecosystems with stochastic sensitivity analysis. - Ecological Modelling 222(14): 25422551.

[32] MacArthur, R. (1955): Fluctuations of animal populations and a measure of community stability. - Ecology 36(3): 533-536.

[33] Marcogliese, D. J., Cone, D. K. (1997): Food webs: a plea for parasites. - Trends in Ecology and Evolution 12: 320-325.

[34] Marcogliese, D. J. (2003): Food webs and biodiversity: are parasites the missing link? Journal of Parasitology 82(S): 389-399.

[35] May, R. M. (1973): Stability and Complexity in Model Ecosystems. - Princeton University Press, Chicago.

[36] McCallum, H., Dobson, A. (1995): Detecting disease and parasite threats to endangered species and ecosystems. - Trends in Ecology and Evolution 10(5): 190-194.

[37] McCann, K. S. (2000): The diversity-stability debate. - Nature 405(6783): 228-233.

[38] Memmott, J., Martinez, N. D., Cohen, J. E. (2000): Predators, parasitoids and pathogens: species richness, trophic generality and body sizes in a natural food web. - Journal of Animal Ecology 69: 1-15.

[39] Michalska-Smith, M. J., Sander, E. L., Pascual, M., Allesina, S. (2018): Understanding the role of parasites in food webs using the group model. - Journal of Animal Ecology 87(3): 790-800. 
[40] Montoya, J. M., Sole, R. V. (2003): Topological properties of food webs: from real data to community assembly models. - Oikos 102: 614-622.

[41] Montoya, J. M., Pimm, S. L., Sole, R. V. (2006): Ecological networks and their fragility. - Nature 442: 259-264.

[42] Mouritsen, K. N., Poulin, R., McLaughlin, J. P., Thieltges, D. W. (2011): Food web including metazoan parasites for an intertidal ecosystem in New Zealand. - Ecology 92(10): 2006

[43] Navia, A. F., Cortes, E., Mejia-Falla, P. A. (2010): Topological analysis of the ecological importance of elasmobranch fishes: A food web study on the Gulf of Tortugas, Colombia. - Ecological Modeling 221(24): 2918-2926.

[44] Nedorezov, L. V. (2012): Continuous-discrete model of population dynamics with time lag in a reaction of intra-population self-regulative mechanisms. - Network Biology 2(4): 139-147.

[45] Nuwagaba, S., Hui, C. (2015): The architecture of antagonistic networks: Node degree distribution, compartmentalization and nestedness. - Computational Ecology and Software 5(4): 317-327.

[46] Okamoto, K., Chen, W., Li, X. Y. (2008): Ranking of closeness centrality for large-scale social networks. - International Workshop on Frontiers in Algorithmics, Springer, Berlin, Heidelberg: 186-195.

[47] Pascual, M., Dunne, J. A. (2006): From small to large ecological networks in a dynamic world. - Ecological Networks: Linking Structure to Dynamics in Food Webs, Oxford University Press, Oxford: 3-24.

[48] Pimm, S. L., Lawton, J. H. (1980): Are food webs divided into compartments? - Journal of Animal Ecology 49: 879-898.

[49] Pimm, S. L. (1991): The balance of nature? Ecological issues in the conservation of species and communities. - University of Chicago Press, Chicago.

[50] Pimm, S. L., Lawton, J. H., Cohen, J. E. (1991): Food web patterns and their consequences. - Nature 350(6320): 669-674.

[51] Price, P. W. (1980): Evolutionary Biology of Parasites (Vol. 15). - Princeton University Press, Chicago.

[52] Sato, T., Egusa, T., Fukushima, K., Oda, T., Ohte, N., Tokuchi, N., Watanabe, K., Kanaiwa, M., Murakami, I., Lafferty, K. D. (2012): Nematomorph parasites indirectly alter the food web and ecosystem function of streams through behavioural manipulation of their cricket hosts. - Ecology Letters 15(8): 786-793.

[53] Shakil, M., Wahab, H. A., Naeem, M., Bhatti, S., Shahzad, M. (2015): The modeling of predator-prey interactions. - Network Biology 5(2): 71-81.

[54] Shams, B., Khansari, M. (2019): Average reachability: A new metric to estimate epidemic growth considering the network structure and epidemic severity. - Network Biology 9(3): 42-57.

[55] Thieltges, D. W., Reise, K., Mouritsen, K. N., McLaughlin, J. P., Poulin, R. (2011): Food web including metazoan parasites for a tidal basin in Germany and Denmark. - Ecology 92(10): 2005.

[56] Wahab, H. A., Ullah, R., Bhatti, S., Shahzad, M., Naeem, M., Hussain, F., Ahmad, S. (2017): An approximate solution for a generalized Hirota-Satsom coupled (Kdv): equation. - Computational Ecology and Software 7(1): 28-37.

[57] Warren, P. H. (1994): Making connections in food webs. - Trends in Ecology and Evolution 9: 136-141.

[58] Warren, C. P., Pascual, M., Lafferty, K. D., Kuris, A. M. (2010): The inverse niche model for food webs with parasites. - Theoretical Ecology 3(4): 285-294.

[59] Wasserman, S. S. (1977): Random directed graph distributions and the triad census in social networks. - Journal of Mathematical Sociology 5(1): 61-86.

[60] Wasserman, S., Faust, K. (1994): Social Network Analysis: Methods and Applications (Vol. 8). - Cambridge University Press, Cambridge. 
[61] Williams, R. J., Martinez, N. D. (2000): Simple rules yield complex food webs. - Nature 404(6774): 180-183.

[62] Winemiller, K. O., Polis, G. A. (1996): Food Webs: Integration of Patterns and Dynamics. - Chapman and Hall, New York: 1-22.

[63] Wood, M. J. (2007): Parasites entangled in food webs. - Trends in Parasitology 23: 8-10.

[64] Zander, C. D., Josten, N., Detloff, K. C., Poulin, R., McLaughlin, J. P., Thieltges, D. W. (2011): Food web including metazoan parasites for a brackish shallow water ecosystem in Germany and Denmark. - Ecological Archives 92(10): 2007.

[65] Zhang, W. J. (2012a): Computational Ecology: Graphs, Networks and Agent-based Modeling. - World Scientific, Singapore.

[66] Zhang, W. J. (2012b): How to construct the statistic network? An association network of herbaceous plants constructed from field sampling. - Network Biology 2(2): 57-68.

[67] Zhang, W. J. (2012c): Several mathematical methods for identifying crucial nodes in networks. - Network Biology 2(4): 121-126.

[68] Zhang, W. J. (2015): A generalized network evolution model and self-organization theory on community assembly. - Selforganizology 2(3): 55-64.

[69] Zhang, W. J. (2016): Network robustness: Implication, formulization and exploitation. Network Biology 6(4): 75-85.

[70] Zhang, W. J. (2018): Fundamentals of Network Biology. - World Scientific Europe, London. 